\title{
Nanoquímica de semicondutores
}

\section{Introdução}

A investigação envolvendo materiais nanocristalinos teve um desenvolvimento notável durante as últimas décadas. Actualmente encontram-se publicados diversos estudos que provam a ocorrência de alterações significativas nas propriedades ópticas, electrónicas e mecânicas de um material quando este passa da forma macrocristalina a um material constituído por partículas de dimensões nanométricas (1-20 nm).[1-5] A síntese química e a caracterização de nanocristalitos inserem-se em um domínio pluridisciplinar frequentemente designado como Nanociências. Embora a miniaturização de dispositivos (ou máquinas) a uma escala nanométrica seja hoje uma realidade, é importante clarificar que muitas das ideias avançadas neste domínio pertencem ao campo ficcional.[6] A engenharia destes sistemas mesoscópicos deu-se o nome de Nanotecnologia sendo apontada como das áreas tecnológicas mais relevantes deste século. 0 advento do microscópio de força atómica e do microscópio de efeito de túnel, em plena década de 80 , vieram estabelecer em definitivo a possibilidade de manipulação de objectos à escala atómica, permitindo a construção de arquitecturas atómicas espantosas, como por exemplo um cercado quântico formado por 48 átomos de ferro formando um círculo com $7.3 \mathrm{~nm}$ de raio, numa superfície de cobre.[7] Estes instrumentos providenciaram os "olhos" e os "dedos" requeridos para a manipulação e caracterização de nanoestruturas. Simulta- neamente, o desenvolvimento paralelo da expansão da capacidade computacional permitiu simulações sofisticadas do comportamento do material a nível nanométrico.

0 físico americano e Prémio Nobel Richard Feynman (1918-1988) na palestra intitulada There is Plenty of Room at the Bottom proferida em 1959,[8] antecipou o aparecimento de grandes descobertas, como por exemplo o fabrico de computadores quânticos que seria teoricamente possivel à luz dos conhecimentos da Física, bastando apenas para tal fabricar materiais e dispositivos à escala atómica/molecular. Hoje, a investigação em computadores quânticos é uma realidade adivinhando-se uma tecnologia computacional de natureza diferente da actualmente existente. Por exemplo, o uso de nanodispositivos pode tornar possivel o armazenamento de toda a informação contida em 1000 CD's em algo com dimensões de um simples relógio de pulso. Por outro lado, os cientistas podem actualmente prever o uso de monocamadas de nanopartículas para uma nova geração de sensores químicos e biológicos, como para comutadores para melhorar/aumentar a capacidade de armazenamento de computadores por um factor de um milhão.

No campo da Química, a preparação de partículas coloidais de dimensões reduzidas remonta ao tempo de Michael Faraday (1791-1867), na altura investigando as propriedades ópticas de colóides de ouro quase monodispersos. No entanto, estudos sistemáticos de síntese $\mathrm{e}$ caracterização de nanocristais de semicondutores são relativamente recentes.[1-5] Este facto é curioso uma vez que a Química se dedica precisamente ao estudo da natureza de construções moleculares. Como adiante se verá, para um químico, um nanocristal de um determinado semicondutor ou metal pode ser entendido como uma macromolécula que apresenta determinadas propriedades decorrentes das dimensões apresentadas. Encontram-se publicados vários artigos de revisão sobre a síntese e propriedades de nanopartículas que dão uma perspectiva actualizada dos estudos realizados na área da nanoquímica.[1-5] Neste artigo salientam-se alguns tópicos de investigação em nanoquímica, com especial ênfase na síntese e propriedades de nanoparticulas semicondutoras com dimensões tipicamente entre 1 e 20 nm (figura 1).

\section{Efeitos quânticos de dimensão em partículas semicondutoras}

Um nanocristal de um semicondutor tem um tamanho intermédio entre a molécula e o composto macrocristalino. As propriedades físicas de partículas com estas dimensões são significativamente diferentes das do semicondutor macrocristalino, fundamentalmente por duas razões:

i) o tamanho da nanopartícula é da ordem de grandeza do comprimento de onda de De Broglie associado ao electrão no semicondutor; 


\begin{tabular}{|l|l|}
\hline $\begin{array}{l}\text { Chip de um circuito } \\
\text { integrado }\end{array}$ & $10 \mathrm{~mm}$ \\
\hline Grão de areia & $1 \mathrm{~mm}$ \\
\hline $\begin{array}{l}\text { Diâmetro de um } \\
\text { fio de cabelo }\end{array}$ & $100 \mu \mathrm{m}$ \\
\hline $\begin{array}{l}\text { Transistor num } \\
\text { circuito integrado }\end{array}$ & $10 \mu \mathrm{m}$ \\
\hline $\begin{array}{l}\text { Partícula de fumo } \\
\text { Largura da molécula } \\
\text { de ADN }\end{array}$ & $1 \mu \mathrm{m}$ \\
\hline $\begin{array}{l}\text { Nanação C-C } \\
\text { nanolécula de etino } \\
\text { contendo cerca de } \\
\text { do } 000 \text { átomos }\end{array}$ & $100 \mathrm{~nm}$ \\
\hline
\end{tabular}

figura 1 Escala das dimensões aproximadas de diversos objectos.

ii) existe uma elevada percentagem de átomos à superfície da partícula comparativamente a um composto macrocristalino.

A observação de um aumento efectivo do hiato óptico de um semicondutor $\left(E_{\mathrm{g}}\right)$ com a diminuição do tamanho de partícula constitui um dos aspectos mais estudados em nanocristalitos semicondutores. $\mathrm{O}$ aumento de $\mathrm{E}_{\mathrm{g}}$ para partículas de dimensões nanométricas é resultado de um efeito do confinamento quântico tridimensional do electrão (e da lacuna) devido às dimensões do nanocristalito; essas partículas têm sido, por isso mesmo, designadas por pontos quânti$\cos$ (quantum dots).[1-5]

Considere-se um determinado semicondutor no qual se faz incidir um fotão com frequência $v=E_{g} / h$, portanto capaz de excitar um electrão para a banda de condução deixando uma lacuna na banda de valência; o electrão e lacuna formam neste caso um excitão de Wannier. Em um semicondutor macrocristalino a atracção de tipo Coulomb entre o par de transportadores de carga que constituem o excitão pode ser negligenciada. Num nanocristal, no entanto, esta atracção não pode ser desprezada visto o excitão se encontrar confinado espacialmente, dadas as reduzidas dimensões da partícula. Utilizando o modelo da partícula na caixa, Brus deduziu a equação 1 que relaciona a energia da primeira transição electrónica do excitão (ou o hiato óptico do semicondutor) com as dimensões do nanocristal. [2]

onde:

$$
\Delta E=\frac{\hbar^{2} \pi^{2}}{2 R^{2}}\left[\frac{1}{m_{\mathrm{e}}}+\frac{1}{m_{\mathrm{h}}}\right]-\frac{1,8 \mathrm{e}^{2}}{\varepsilon R}
$$

$\Delta E=$ desvio relativamente ao valor de $E_{g}$ do material macrocristalino; $\hbar=$ constante de Planck; $R=$ dimensão característica do nanocristal (raio); $m_{\mathrm{e}}=$ massa efectiva do electrão; $m_{\mathrm{h}}=$ massa efectiva da lacuna; $e=$ carga elementar; $\varepsilon=$ permitividade.

A equação 1 é o resultado de um modelo relativamente simples e com várias limitações, permitindo, no entanto, ad-

\section{figura 2}

a) Evolução do hiato óptico de um semicondutor com a variação do tamanho de partícula; b) amostras de nanocristais de CdSe (dispersos em matriz orgânica) com tamanhos médios de partícula diferentes (2 a $5 \mathrm{~nm}$ de diâmetro).

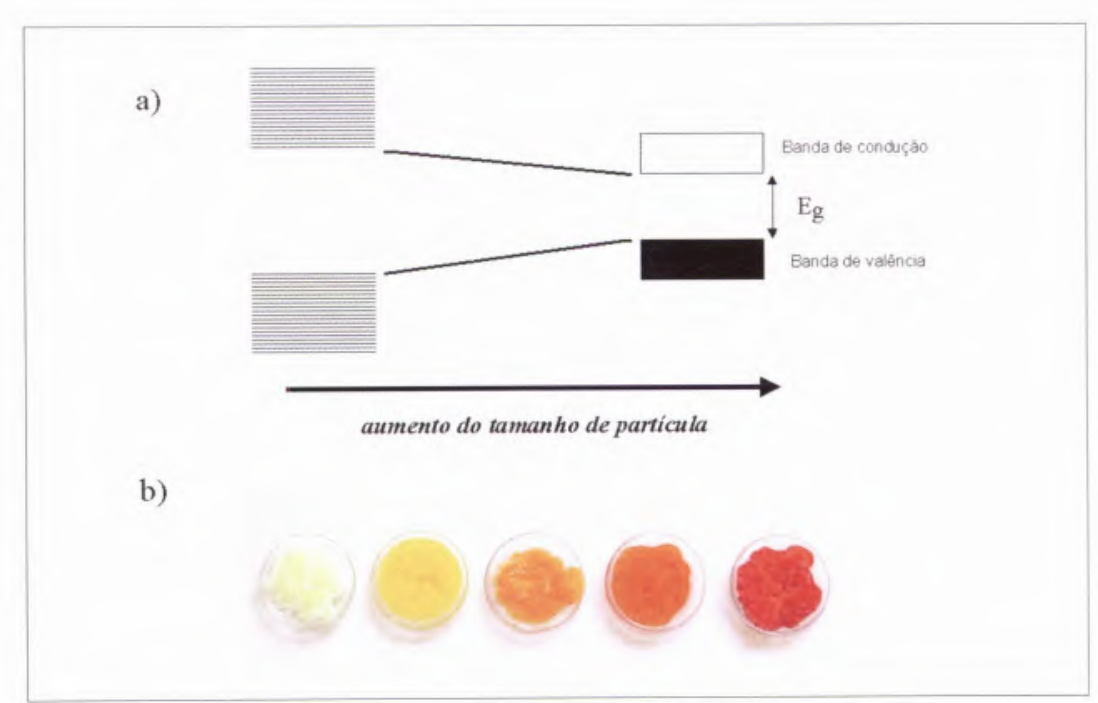

quirir um conhecimento qualitativo do efeito de confinamento quântico em partículas semicondutoras. Esta equação torna-se particularmente útil para o químico de sintese pois permite rapidamente fazer uma estimativa do tamanho de partícula médio utilizando os valores de absorção óptica obtidos experimentalmente. No entanto, e para partículas demasiado pequenas $(<3 \mathrm{~nm}$ ), o uso da equação 1 conduz a resultados que se desviam consideravelmente da realidade. Repare-se que segundo a equação 1 , para um determinado semicondutor existe um valor de $\mathrm{R}$ abaixo do qual o hiato óptico do semicondutor aumenta à medida que o tamanho de partícula diminui (figura 2). Em alguns semicondutores, como por exemplo o CdSe, a diminuição do tamanho médio de partícula e o consequente aumento de $E_{g}$, torna-se evidente pela simples observação da cor da amostra (figura 2). o CdSe macrocristalino em pó apresenta uma cor castanha escura enquanto no CdSe nanocristalino a cor pode variar do amarelo ao vermelho, dependendo do tamanho médio de partícula.

Para o semicondutor CdS, por exemplo, são prevísiveis efeitos de confinamento quântico para CdS policristalino com um diâmetro médio de partícula de 6 $\mathrm{nm}$ ou inferior. Estes efeitos quânticos de dimensão têm sido observados para outros semicondutores nanocristalinos 
como por exemplo CdSe,[9] PbS[10]; $\mathrm{PbSe}[11], \operatorname{InP}[12], \mathrm{Cd}_{3} \mathrm{P}_{2}$ [13], etc.

No entanto semicondutores com estruturas cristalinas mais complexas, como por exemplo os de estrutura lamelar $\left(\mathrm{MoS}_{2}, \mathrm{Bi}_{2} \mathrm{~S}_{3}, \mathrm{Pbl}_{2}\right.$, etc), têm sido menos investigados e é evidente na literatura especializada o reduzido número de métodos químicos reprodutiveis que originam nanocristais de qualidade elevada destes semicondutores. [14-16]. Neste tipo de materiais verifica-se que o crescimento de partícula ocorre de uma forma anisotrópica não sendo correcto aplicar a equação 1 na estimativa do hiato óptico do semicondutor nanocristalino. Para os nanocristais de estrutura lamelar foi proposto um modelo para o confinamento quântico dos transportadores de carga que tem em conta a anisotropia da nanoestrutura, equação 2 [14]:

$\Delta E \cong \frac{\hbar^{2} \pi^{2}}{2 \cdot \mu_{\mathrm{xy}}}\left(\frac{2}{\mathrm{~L}^{2}{ }_{\mathrm{xy}}}\right)+\frac{\hbar^{2} \pi^{2}}{2 \cdot \mu_{z}}\left(\frac{1}{\mathrm{~L}^{2}{ }_{z}}\right)$

Em que: $x, y=$ coordenadas do plano lamelar da partícula; $z$ = coordenada perpendicular ao plano lamelar; $\mu_{i}=$ massas efectivas reduzidas do par electrão-lacuna segundo os planos mencionados; $L_{i}=$ dimensão da partícula segundo a direcção i; $\Delta E=$ energia da 1. ${ }^{a}$ transição electrónica do excitão; $\hbar=$ constante de Planck.

\section{Propriedades ópticas e estrutura}

Devido ao efeito de confinamento quântico, e para uma mesma temperatura, o espectro de absorção óptica de uma amostra constituída por nanocristalitos semicondutores evidencia um desvio, para maiores energias, do limite superior de absorção relativamente ao valor típico do semicondutor macrocristalino (figura 3). Este facto experimental tem sido reproduzido para uma série de semicondutores nanocristalinos preparados segundo técnicas de síntese diversas; constitui por isso uma das evidências mais directas do aumento do hiato óptico do semicondutor com a di-

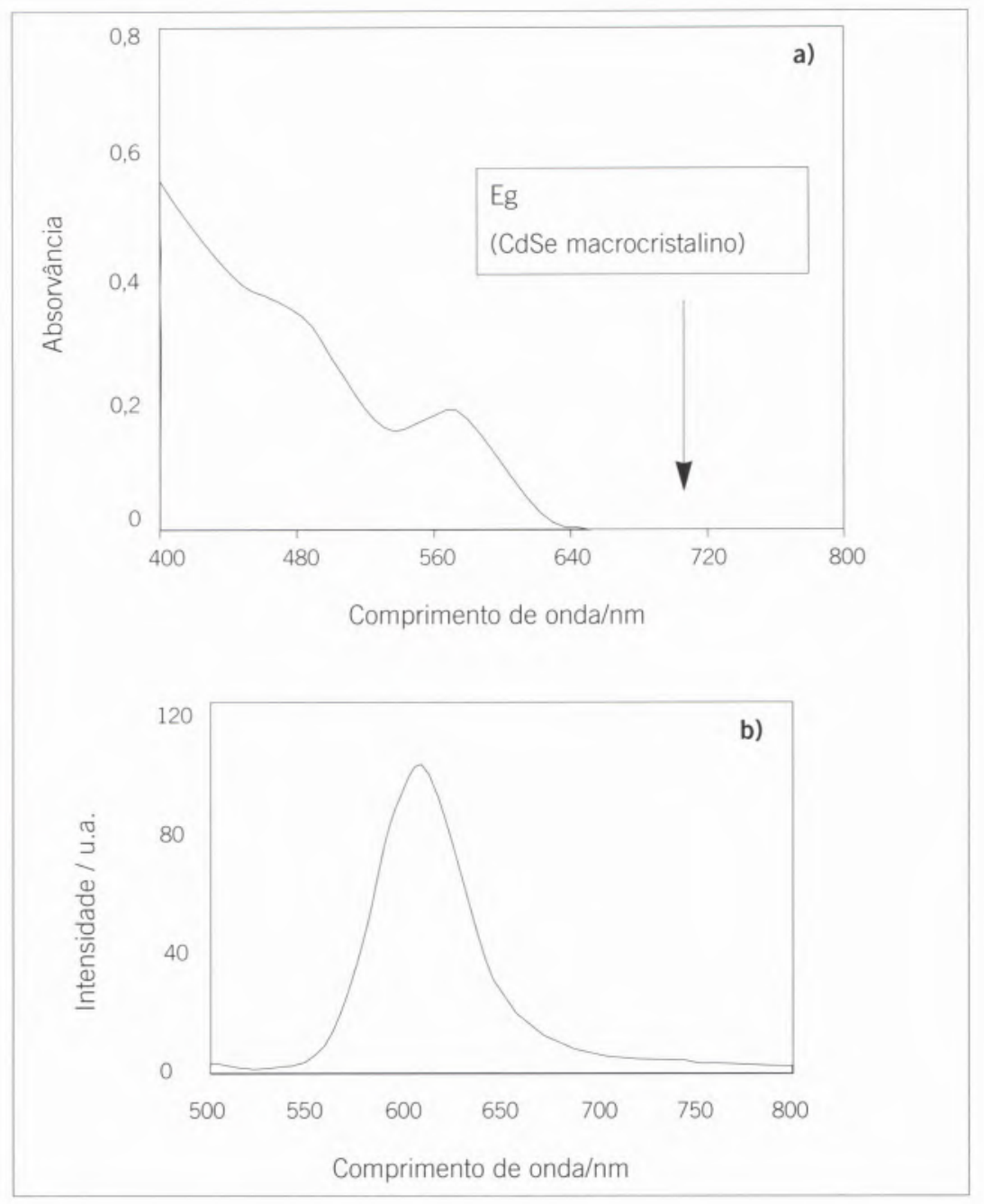

figura 3 Espectros ópticos de nanocristais de CdSe $\left(d_{m} \approx 5 \mathrm{~nm}\right)$ dispersos em tolueno: a) absorção no visivel ; b) fotoluminescência $\left(\lambda_{\text {exc }}=465 \mathrm{~nm}\right)$.

minuição do tamanho de partícula (figura 2). Em amostras constituídas por nanocristais com uma dispersão de tamanhos reduzida é possível a observação de um máximo de absorção (pico excitónico) associado à primeira transição electrónica do excitão. 0 aparecimento de estrutura no espectro óptico de um semicondutor nanocristalino sugere a presença de conjuntos de níveis de energia discretos. No caso limite de agregados (ou clusters), as partículas do semicondutor são de facto nanoestruturas de natureza molecular, com diâmetros tipicamente inferiores a $2 \mathrm{~nm}$; embora partículas com dimensões nesta ordem de grandeza não sejam objecto de atenção especial neste artigo, existe actualmente um interesse muito especial na sua investigação pois é neste estádio que ocorre a transição da molécula para sólido cristalino.[17] Para além disso, estes agregados, por exemplo os tiolatos de metais ou os polioxometalatos, podem actuar como unidades químicas no fabrico de nanoestruturas de maiores dimensões. $[17,18]$ No caso de moléculas de polioxometalatos de grandes dimensões,[18] a própria molécula corresponde a uma nanopartícula tratando-se assim de materiais nanocristalinos verdadeiramente monodispersos. É o caso do composto $\left[\left\{(\mathrm{Mo}) \mathrm{Mo}_{5} \mathrm{O}_{2}\left(\mathrm{H}_{2} \mathrm{O}\right)_{4} \mathrm{CH}_{3} \mathrm{COO}\right\}_{12}\left[\mathrm{MoO}\left(\mathrm{H}_{2}\right.\right.\right.$ O) $\}_{30}$ ] (figura 4), cuja estrutura é composta por 12 unidades pentagonais do tipo $\left\{(\mathrm{Mo}) \mathrm{Mo}_{5}\right\}$ ligadas por intermédio de 30 pontes de $\mathrm{O}=\mathrm{Mo}\left(\mathrm{H}_{2} \mathrm{O}\right)$. Os iões de molibdénio estão localizados no centro de octaedros, com excepção do molibdénio central em cada unidade pentagonal que se encontra no centro de uma bipirâmide pentagonal. 
A aplicação de técnicas de difracção de raios $X$ ou de electrões em semicondutores nanocristalinos, demonstra que estruturalmente os nanocristais podem ser entendidos como fragmentos da rede cristalina do composto semicondutor em causa. Por exemplo, a figura 5 , mostra imagens de microscopia electrónica por transmissão de diversos nanocristais, em que a estrutura cristalina para cada amostra é a mesma que é verificada nos semicondutores macrocristalinos respectivos, nas mesmas condições de temperatura e pressão. Repare-se como as nanopartículas apresentam uma morfologia variável de semicondutor para semicondutor, embora haja uma homogeneidade morfológica para a mesma amostra. Este é um aspecto relevante na preparação de nanocristais de qualidade elevada e em que alguns métodos químicos de síntese têm tido sucesso.

A estrutura cristalina interna e a estrutura electrónica de diversos semicondutores nanocristalinos encontram-se razoavelmente compreendidas. O mesmo não se pode afirmar relativamente à natureza da superfície dos nanocristais. Em um material nanocristalino, e devido à existência de uma área de superfície elevada, é de prever que propriedades dependentes da natureza da superfície sejam significativamente diferentes das encontradas no material macrocristalino. Por exemplo, a adsorção de moléculas orgânicas à superfíce da partícula causa uma perturbação nos níveis energéticos superficiais, podendo modificar as propriedades ópticas do sistema. Uma dessas propriedades é a luminescência exibida por semicondutores nanocristalinos após irradiação com fotões

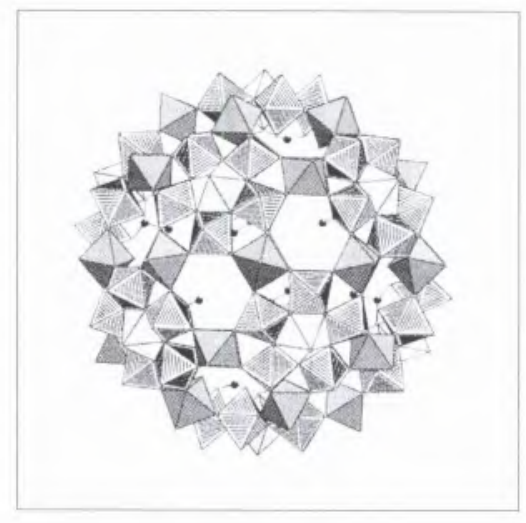

figura 4 Representação polièdrica da estrutura do poli-oxometalato.

$\left[\left\{(\mathrm{Mo}) \mathrm{Mo}_{5} \mathrm{O}_{2}\left(\mathrm{H}_{2} \mathrm{O}\right)_{4} \mathrm{CH}_{3} \mathrm{COO}\right]_{12}\left\{\mathrm{MoO}\left(\mathrm{H}_{2} \mathrm{O}\right)\right\}_{30}\right]$ Cortesia do Professor A. Muller e Wiley-VCH [18])

de energia superior a $E_{g}$. A fotoluminescência de CdS nanocristalino tem sido especialmente estudada.[19] Os defeitos de rede no CdS macrocristalino, nomeadamente vagas de sulfureto, actuam como locais de recombinação não-radiativa dos transportadores de carga; o espectro de luminescência deste sólido apresenta normalmente uma banda de emissão larga que se estende até à região do vermelho. No caso do semicondutor nanocristalino esse tipo de defeitos de rede localizam-se sobretudo à superfície das partículas. Contudo, quando a superfície é passivada com moléculas orgânicas, por exemplo com moléculas do solvente utilizado na síntese, a emissão a menor energia é atenuada. Nesta situação detecta-se luminescência, à temperatura ambiente, a um comprimento de onda próximo do $E_{\mathrm{g}}$ do semicondutor nanocristalino. 0 CdSe apresenta um comportamento análogo ao do CdS, no entanto exibe fotoluminescência numa gama de comprimentos de onda mais larga. A Figura
3 apresenta o espectro de emissão de uma amostra de CdSe nanodisperso em tolueno e o respectivo espectro de absorção óptica.

Na figura 3-b, a banda de emissão a $610 \mathrm{~nm}$, ligeiramente desviada para o vermelho em relação ao pico excitónico no espectro de absorção (figura 3-a), tem sido associada à recombinação (quase) excitónica no CdSe nanocristalino. [2,9] O revestimento dos nanocristais com uma segunda fase inorgânica, em substituição de moléculas orgânicas, tem resultado numa maior intensidade da emissăo excitónica; por exemplo no caso do CdS e CdSe têm sido utilizados, com sucesso, o $\mathrm{Cd}(\mathrm{OH})_{2}$ e o $\mathrm{ZnS}$ respectivamente, como fases passivantes. $[19,20]$ À semelhança do que é observado no espectro de absorção óptica, a diminuição do tamanho médio das partículas do semicondutor desvia o máximo de emissão para maiores energias devido ao efeito de confinamento quântico.

\section{Métodos de síntese}

Um sistema nanocristalino ideal deveria ser constituido por partículas cristalinas monodispersas, com uma composição química homogénea ao nível molecular e com a superfície uniformemente passivada. A preparação de um sistema com estas características constitui um verdadeiro desafio; de facto, a investigação de novas estratégias de sintese de semicondutores nanocristalinos é actualmente um tópico de intensa investigação na área dos novos materiais. Embora não seja aqui abordado em especial, refirase a preparação química de silício nanocristalino; o silício é o semicondutor tecnologicamente mais relevante tendo-se

figura 5 Imagens obtidas por microscopia electrónica de transmissão de nanocristais de semicondutores: a) PbS, a seta indica o efeito da sobreposição de nanocristais (barra $=10 \mathrm{~nm}) ;$ b) CdSe (barra $=10 \mathrm{~nm}) ;$ c) $\mathrm{Cd}_{3} \mathrm{P}_{2}($ barra $\left.=5 \mathrm{~nm}) ; \mathrm{d}\right) \mathrm{Bi}_{2} \mathrm{~S}_{3}($ barra $=70 \mathrm{~nm})$.
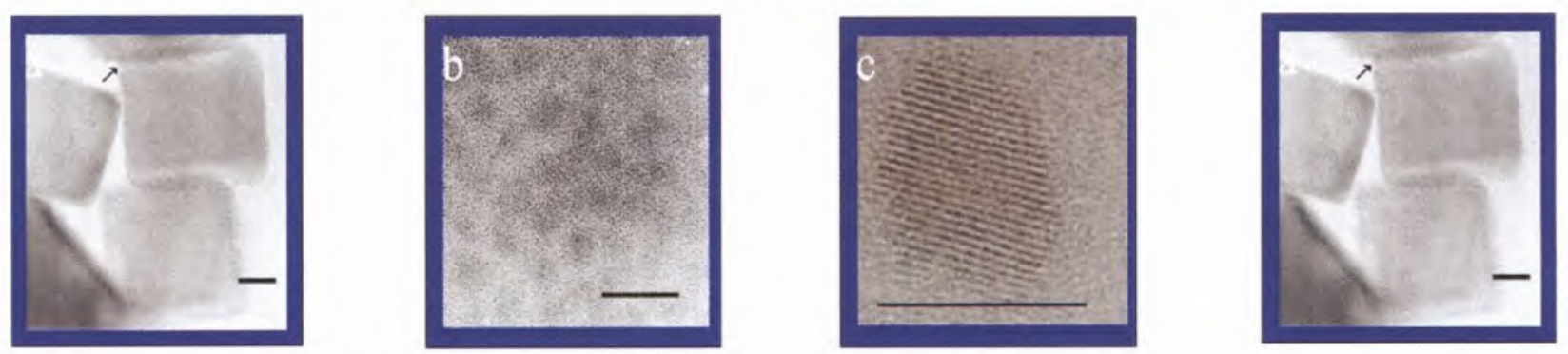
observado recentemente fenómenos de luminescência no silício poroso.[21]

Os semicondutores nanocristalinos têm sido preparados por métodos químicos muito diversos. Dos métodos em fase liquida existentes serão referidos, embora de um modo sucinto, os mais utilizados. Os métodos de precipitação controlada com vista à formação de um colóide estável têm sido dos mais investigados e são conhecidos desde há já muito tempo. Neste campo, é de realçar o trabalho de Weller e Henglein [1,4]; estes autores prepararam nanocristalitos de diversos sistemas, como por exemplo CdS, PbS, $\mathrm{Ag}_{2} \mathrm{~S}, \mathrm{Cd}_{3} \mathrm{P}_{2}$ e $\mathrm{Zn}_{3} \mathrm{P}_{2}$, pela adição a uma solução do catião respectivo, de um composto contendo o não metal. Os nanocristalitos formados são impedidos de aglomerar e coalescer, pela presença de agentes estabilizadores como sejam polifosfatos ou polímeros orgânicos. Não obstante a sua aparente simplicidade os métodos de precipitação controlada exigem rigor no controlo dos vários parâmetros experimentais envolvidos. A ocorrência de pequenas alterações nas condições experimentais conduz invariavelmente ao aparecimento de nanocristais com diferente tamanho médio e, portanto, com propriedades ópticas diferentes. De entre os parâmetros experimentais mais relevantes destacam-se a natureza do solvente, a temperatura de reacção e a concentração dos reagentes.

A formação controlada do semicondutor nanocristalino pode também ser conseguida utilizando materiais que funcionam como nano-compartimentos para a reacção de precipitação. Estes métodos, quando comparados com métodos de precipitação directa, permitem um maior controle no tamanho das partículas bem como uma maior estabilidade contra processos de aglomeração. A preparação de ZnS em microemulsões [22] ou a formação de agregados de CdS em zeólitos [23] são exemplos deste tipo de métodos. Faz-se notar que o tamanho de partícula é, no primeiro caso, controlado pelas dimensões da gota (razão molar água/surfactante) e, no segundo caso, pelas dimensões dos poros do zeólito.
Dado o seu elevado interesse tecnológico, refira-se ainda a preparaçăo de semicondutores nanocristalinos utilizando precursores moleculares. Estes métodos possibilitam a preparação de semicondutores que não são obtidos facilmente pela adição controlada de um não metal a um catião metálico. Podem constituir também uma alternativa, aos métodos em solução aquosa, quando é crucial evitar a incorporação de iổes óxido na rede do semicondutor. Por outro lado, ao utilizar temperaturas relativamente elevadas torna-se um método bastante atractivo para obtenção de nanocristais, por exemplo de semicondutores III-V, que não cristalizam facilmente. A preparação de nanocristalitos de CdSe, por exemplo, envolve precisamente a reacção, a temperatura elevada $\left(250^{\circ} \mathrm{C}\right)$, de $\mathrm{Cd}\left(\mathrm{CH}_{3}\right)_{2}$ e TOPSe utilizando TOPO (óxido de trioctilfosfina) como solvente. [9] Este método apresenta, neste caso concreto, a grande desvantagem de ser necessária a manipulação de um composto pirofórico, como é o caso do $\mathrm{Cd}\left(\mathrm{CH}_{3}\right)_{2}$, a temperaturas elevadas.

Esta limitação é contudo ultrapassada pela substituição dos referidos reagentes por precursores unimoleculares.[24] Estes compostos, como por exemplo o precursor $\mathrm{MeCd}\left(\mathrm{Se}_{2} \mathrm{CNEt}_{2}\right)_{2}\left(\mathrm{Me}=\mathrm{CH}_{3}\right.$; $\mathrm{Et}=\mathrm{C}_{2} \mathrm{H}_{5}$ ), permitem a sintese de nanocristais numa etapa única, pela injecção no vaso de reacção do metal e não metal que se encontram ligados quimicamente no precursor (equação 3).

$\mathrm{MeCd}\left(\mathrm{Se}_{2} \mathrm{CNEt}_{2}\right)_{2} \stackrel{2000 \mathrm{C}}{\rightarrow} \mathrm{CdSe}+\underset{\text { produtos }}{\text { outros }}$

(3)

Independentemente do tipo de precursor usado, $\mathrm{CdMe}_{2} / \mathrm{TOPSe}$ ou MeCd $\left(\mathrm{Se}_{2} \mathrm{CNEt}_{2}\right)_{2}$, o factor temperatura revela-se crucial no controlo do tamanho das partículas de CdSe, aumentando este com o aumento da temperatura (figura 2).

Os métodos referidos anteriormente podem ser utilizados conjuntamente e de uma forma complementar. Por exemplo, os precursores moleculares $\mathrm{ZnEt}_{2}$ e $\mathrm{Se}\left(\mathrm{SiMe}_{3}\right)_{2}$ reagem quimicamente no interior de microemulsōes invertidas, originando nanocristais de
ZnSe com morfologia definida e superficie passivada pelas moléculas de surfactante (equação 4).

$$
\mathrm{ZnEt}_{2}+\mathrm{Se}\left(\mathrm{SiMe}_{3}\right)_{2} \stackrel{-2 \mathrm{SiEtMe}_{3}}{\longrightarrow} \mathrm{ZnSe}
$$

\section{Aplicações de nanocristalitos de semicondutores}

Referem-se nesta secção algumas aplicações relevantes para materiais nanocristalinos. Ao nível da indústria de electrónica a preparação de nanocristalitos de semicondutores para fins tecnológicos acompanha a tendência de miniaturização de produtos, tal como se tem verificado no fabrico de chips para computadores. O grupo de Alivisatos da Universidade de Berkeley [25] descreveu o fabrico de um dispositivo electroluminescente à base de um material hibrido inorgânico/orgânico contendo nanocristalitos de CdSe numa base polimérica. A luminescência na gama do vermelho ao amarelo é consequência do efeito de confinamento quântico observado em nanocristalitos de CdSe com diferentes distribuições de tamanhos; a luminescência na zona do verde é atribuída ao polímero. Pelo facto de a luminescência observada neste sistema ser dependente da voltagem aplicada é de realçar a sua utilização no fabrico de monitores planos policromáticos. Também nanocristais de CdSe passivados com silica e com distribuições de tamanho distintas estão a ser investigados como sondas biológicas luminescentes.[26] A utilização de nanoestruturas semicondutoras associadas a sistemas biológicos será porventura umas das áreas onde se prevêm avanços significativos num futuro próximo.

Existem compostos que podem ocorrer como nanomateriais e que são actualmente comercializados, como por exemplo os zeólitos utilizados em catálise ou os materiais cerâmicos finamente divididos. Os estudos de catálise utilizando sistemas nanocristalinos semicondutores são no entanto relativamente recentes.[27] Este tipo de materiais apresenta uma elevada área de superficie sendo a sua investigação bastante promissora no domínio da catálise. Por exemplo, a preparação fotoquímica de 
ácido fórmico a partir de soluções aquosas saturadas em $\mathrm{CO}_{2}$ na presença de CdSe nanocristalino ( $50 \AA$ ); i esta reacção não ocorre quando se utilizam sistemas constituidos por partículas de CdSe de maiores dimensōes.[27] A capacidade foto-oxidante do CdSe nanocristalino resulta do efeito de confinamento quânti$\mathrm{co}$, existindo nesse caso níveis energéticos favoráveis a receberem electrōes de espécies redutoras à superfície do semicondutor.

O trabalho de Grätzel e colaboradores, tem demonstrado a elevada eficiência de células foto-electroquímicas contendo $\mathrm{TiO}_{2}$ nanocristalino como eléctrodo de suporte [3]. A área superficial elevada destes eléctrodos permite um contacto mais eficiente entre o sensibilizador e o semicondutor. Os sensibilisado res utilizados têm sido geralmente corantes ou complexos metálicos fotoquimicamente activos dos quais se destacam complexos de ruténio. [3] A utilização de nanocristalitos semicondutores como sensibilisadores encontra-se igualmente descrita. Por exemplo, Weller et al. demonstraram que partículas de nanocompósitos do tipo $\mathrm{CdS} / \mathrm{TiO}_{2}$ apresentam algumas vantagens relativamente à utilização de complexos metálicos ou corantes, pois além de serem mais económicos são quimicamente mais resistentes. [4] 0 desenvolvimento de novos métodos de síntese de compósitos nanocristalinos é actualmente um dos desafios mais interessantes em síntese inorgânica.

Outra área de interesse tecnológico destes materiais é a sua utilização como pigmentos em tintas com propriedades inovadoras. Os nanomateriais podem melhorar as propriedades térmicas, ópticas e mecânicas da matriz polimérica, sem que para tal se verifique um aumento significativo da densidade do compósito, uma vez que as quantidades de carga a utilizar serão reduzidas. Dado que as dimensões de partícula são tipicamente inferiores ao comprimento de onda da luz visivel, o material compósito final mantém a transparência do polímero de base, pelo que estes materiais podem ser entendidos como uma nova classe de pigmentos inorgânicos.

\section{Conclusões}

A investigação de estruturas cristalinas de dimensões nanométricas é actualmente um desafio estimulante. Importa destacar, neste contexto, a importância do desenvolvimento de métodos de síntese de nanomateriais com potencial para aplicaçōes futuras, bem como métodos que permitam, por manipulação, organizar estes materiais em nano-estruturas funcionais. O sucesso da nanoquímica como factor de desenvolvimento social e humano passa necessariamente pela constituição de equipas de investigação interdisciplinares e coerentes, cuja transversalidade de conhecimentos deverá ser entendida como uma mais valia na análise de problemas científicos e tecnológicos claramente identificados. Neste contexto, uma abordagem desta temática ao nivel dos curricula universitários talvez merecesse ser considerada.

\section{Agradecimentos}

O.C.M. agradece à FCT uma bolsa de doutoramento. Agradece-se o financiamento concedido pela FCT no âmbito do projecto POCTI/35458/CTM/2000 comparticipado pelo FEDER.

\section{Referências}

1. Henglein, A. Chem. Rev., 1989, 89, 1861

2. Steigerwald, M. L.; Brus, L. E. Acc. Chem. Res., 1990, 23, 183

3. Hagfeldt, A.; Gratzel M. Chem. Rev., $1995,95,49$

4. Weller, H. Adv. Mater., 1993, 5, 88.

5. Trindade, T.; O Brien, P.; Pickett, N. L. Chem. Mater, no prelo.

6. Nanotechnology: Molecular Speculations on Global Abundance, B. C. Crandal (ed.), The MIT Press, Cambridge, Massachusetts, 1996; Scientific American-Special Nanotechnology Issue, September, 2001.

7. Crommie, M. F; Lutz, C. P.; Eigler, D. M. Science, 1993, 262, 218

8. Feynmam, R. P. Eng. Sci., 1960, 23, 22, (reprinted in J. Micromech. Systems, 1992, $1,60)$.

9. Murray, C. B.; Norris, D. J.; Bawendi, M. G. J. Am. Chem. Soc., 1993, 115, 8706.
10. Gallardo, S.; Gutiérrez, M.; Henglein, A.; Janata, E. Ber. BunsenGes. Phys. Chem., 1989, 93, 1080.

11. Trindade, T.; Monteiro, O. C.; O'Brien, P.; Motevalli, M. Polyhedron, 1999, 18, 1171.

12. Guzelian, A. A.; Katari, J. E. B.; Kadavanich, A. V.; Banin, U.; Hamad, K.; Juban, E.; Alivisatos, A. P.; Wolters, R. H.; Arnold, C.

C.; Heath, J. R. J. Phys. Chem., 1996, 100. 7212.

13. Green, M.; O'Brien, P. Adv. Mater., $1998,10,527$.

14. Variano, B. F.; Hwang, D. M.; Sandroff, C. J.; Wiltzius, P.; Jing T. W.; Ong, N. P. J. Phys. Chem., 1987, 91, 6455.

15. Mdleleni, M. M.; Hyeon, T.; Suslick, K. S. J. Am. Chem. Soc., 1998, 120, 6189.

16. Monteiro, O. C.; Nogueira, H. S.; Trindade, T.; Motevalli, M. Chem. Mater., 2001. 13, 2103.

17. Herron, N.; Calabrese, J. C.; Farneth, W. E.; Wang, Y. Science, 1993, 259, 1426.

18. Müller A.; Shah, S. Q. N.; Bögge, H.; Schmidtmann, M.; Kögerler, P.; Hauptfleisch, B.; Leiding, S.; Wittler, K. Angew. Chem. Int. Ed. 2000, 39, 1614.

19. Spanhel, L.; Haase, M.; Weller, H.; Henglein, A. J. Am. Chem. Soc., 1987, 109, 5649.

20. Dabbousi, B. O.; Rodriguez-Viejo, J.; Mikulec, F. V.; Heine, J. R.; Mattoussi, H.; Ober, R.; Jensen, K. F.; Bawendi, M. G. J. Phys. Chem. B, 1997, 101, 9463.

21. Brus, L. J. Phys, Chem., 1994, 98 , 3575 .

22. Kortan, A. R.; Hull, R.; Opila, R. L.; Bawendi, M. G.; Steigerwald, M. L.; Carrol, P.J.; Brus, L. E. J. Am. Chem. Soc., 1990, 112, 1327.

23. Wang, Y.; Herron, N. J. Phys. Chem., $1988,92,4988$.

24. Trindade, T.; O'Brien, P.; Zhang, $X$. Chem. Mater., 1997, 9, 523.

25. Colvin, V. L.; Schlamp, M. C.; Alivisatos, A. P. Nature, 1994, 370, 354.

26. Bruchez Jr, M.; Moronne, M.; Gin, P.; Weiss, S.; Alivisatos, A. P. Science, 1998, 281, 2013.

27. Nedeljkovic, J. M.; Nenadovic, M. T.; Micic, O. I.; Nozik, A. J. J. Phys. Chem.,

1986, 90, 12. 